Special issue of the International Conference on Computational and Experimental Science and Engineering (ICCESEN 2014)

\title{
One-Loop Perturbative Corrections for Noncommutative $N=1$ Supesymetric Gauge Theories in two Dimensions
}

\author{
K. ZAIBAK AND K. BOUAKAZ*
}

Departement of Physics, Laboratoire de Physique des Particules et de Physique Statistique Ecole Normale Supérieure, BP 92 Vieux-Kouba, Algiers, Algeria

\begin{abstract}
We calculate the contribution of the tadpole for gauge field of the noncommutative gauge supersymmetric theory in two dimensions in Minkowski space. We have found two types of divergences UV/IR similar to those found in the contribution of the ghost field loop.
\end{abstract}

DOI: 10.12693/APhysPolA.128.B-330

PACS: 14.80.Ly, 11.30.Pb, 14.80.Da, 12.60.Jv

\section{Introduction}

Noncommutative field theories possess specific properties of UV- and IR-divergencies which distinguish them from ordinary theories very essentially. It was shown for noncommutative scalar field models $[1,2]$ that the UVdivergencies lead to additional IR-singularities of effective action that was called the UV/IR-mixing. A study of extended supersymmetric model deserves a special attention. For example, maximally extended supersymmetric noncommutative $N=4 \mathrm{SYM}$ is finite and has no UV/IRmixing [3] what is convenient for investigating the lowenergy behavior [4].

Our work deals mainly with the perturbatrive corrections to $N=1$ noncommutative supersymmetric $U^{*}(1)$ gauge theory at one loop order with two dimensions in Minkowki space. We treat the deformation of the gauge theory $U^{*}(1)$ supersymmetric $N=1$ in two dimensions. We quantify the theory to deduce the terms of gauge and ghosts fields of Faddeev Popov. Thereafter, we derive the Feynman rules of this theory, then we calculate the radiative corrections for the gauge field propagator of this theory at one loop order.

The present work gives the contribution of the tadpole for gauge field of the noncommutative gauge supersymmetric theory in two dimensions in Minkowski space.

\section{Noncommutative Gauge Supersymmetric Theory in Two Dimensions}

The noncommutative action of pure $U^{*}(1)$ gauge supersymmetric theory in

Minkowski space time with two dimensions is given by [5]:

$$
S=\int \mathrm{d} x^{2}\left\{-\frac{1}{4} F_{\mu \nu}^{*} F^{\mu \nu}-\frac{1}{2 \alpha}\left(\partial^{\mu} B_{\mu}\right)^{2}-\frac{1}{2}\left(\partial_{\mu} M\right)^{2}\right.
$$

\footnotetext{
*corresponding author; e-mail: kbouakaz@yahoo.fr
}

$$
\begin{aligned}
& -\frac{\mathrm{i}}{2}\left(1-\frac{1}{\alpha}\right) \bar{\lambda} \gamma^{\mu} \partial_{\mu} \lambda-\frac{\mathrm{i}}{2} g B^{\mu *} M * \overleftrightarrow{\partial}_{\mu} M \\
& +\frac{1}{2} g \bar{\lambda} \gamma^{5 *} \lambda^{*} M+\mathrm{i} \bar{c} \partial^{\mu} D_{\mu} c-\frac{1}{2} g \bar{\lambda} \gamma^{\mu *} \lambda^{*} B_{\mu} \\
& \left.+\frac{1}{4} g^{2}\left(B_{\mu}^{*} M^{*} B^{\mu *} M-B_{\mu}^{*} B^{\mu *} M^{*} M\right)\right\},
\end{aligned}
$$

where $F_{\mu \nu}=\partial_{\mu} B_{\nu}-\partial_{\nu} B_{\mu}+\frac{\mathrm{i} g}{2}\left[B_{\mu}, B_{\nu}\right], D_{\mu} M=\partial_{\mu} M+$ $\frac{\mathrm{i} g}{2}\left[B_{\nu}, M\right]_{*}$.

As a result the propagator is the same as in the commutative counterpart but each vertex contain a supplementary phase factor which depends on deformation parameter [5].

\subsection{The contribution of the tadpole for gauge field propagator}

By using the Feynman rules, we obtain the contribution of the tadpole for gauge field propagator (Fig. 1):

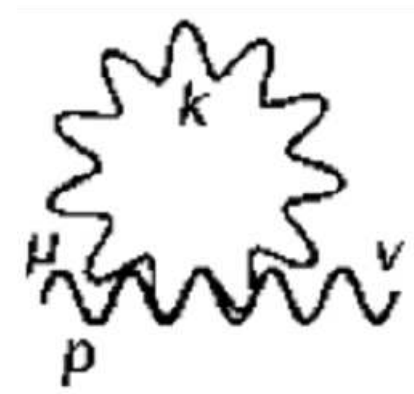

Fig. 1. The tadpole for gauge field propagator.

$$
\pi_{\mu \theta}=\mathrm{i} g^{2} g_{\mu \theta}(\alpha+1) \int \frac{\mathrm{d}^{2} k}{(2 \pi)^{2}} \frac{\sin ^{2} \frac{1}{2}(k \wedge p)}{\left(k^{2}+\mathrm{i} \varepsilon\right)} .
$$

This contribution is composed of planar and nonplanar diagrams:

$$
\pi_{\mu \theta}^{P L}=\frac{1}{2} \mathrm{i} g^{2} g_{\mu \theta}(\alpha+1) \int \frac{\mathrm{d}^{2} k}{(2 \pi)^{2}} \frac{1}{\left(k^{2}-m^{2}+\mathrm{i} \varepsilon\right)},
$$




$$
\begin{aligned}
\pi_{\mu \theta}^{\mathrm{NPL}} & =\frac{1}{2} \mathrm{i} g^{2} g_{\mu \theta}(\alpha+1) \\
& \times \int \frac{\mathrm{d}^{2} k}{(2 \pi)^{2}} \frac{1}{\left(k^{2}-m^{2}+\mathrm{i} \varepsilon\right)} \mathrm{e}^{\mathrm{i} k \rho},
\end{aligned}
$$

where $\rho^{\mu}=\theta^{\mu \nu} p_{\nu}$ and $\rho^{2}=\rho_{0}^{2}-\rho_{1}^{2}$.

Applying the Wick rotation, Schwinger formula and the Gauss integral with modified Bessel function:

$$
\begin{aligned}
& \pi_{\mu \theta}^{(d) P L}=\frac{1}{4 \pi} g^{2} g_{\mu \theta}(\alpha+1) \\
& \quad \times\left(\gamma+\ln \sqrt{\frac{m^{2}}{L^{2}}}+O\left(\frac{m^{2}}{L^{2}} \ln \frac{L}{m}\right)\right) .
\end{aligned}
$$

For the non-planar contribution, the situation is even worse in Minkowski space time due to its indefinite metric [2].

2.1.1. Space like case $\rho^{2}<0$

$$
\begin{aligned}
& \pi_{\mu \theta}^{\mathrm{NPL}}=\frac{1}{4 \pi} g^{2} g_{\mu \theta}(\alpha+1) \\
& \quad \times\left(\gamma+\ln \sqrt{\frac{m^{2}}{L_{1 \mathrm{eff}}}}+O\left(\frac{m^{2}}{L_{1 \mathrm{eff}}} \ln \frac{L_{1 \mathrm{eff}}}{m}\right)\right),
\end{aligned}
$$

where $L_{1 \mathrm{eff}}=\frac{1}{4\left(\rho^{1}\right)^{2}+\frac{1}{L^{2}}}$.

2.1.2. Time like $\rho^{2}>0$

$$
\begin{aligned}
& \pi_{\mu \theta}^{\mathrm{NPL}}=\frac{1}{4 \pi} g^{2} g_{\mu \theta}(\alpha+1) \\
& \quad \times\left(\gamma+\ln \sqrt{\frac{m^{2}}{L_{0 \mathrm{eff}}}}+O\left(\frac{m^{2}}{L_{0 \mathrm{eff}}} \ln \frac{L_{0 \mathrm{eff}}}{m}\right)\right),
\end{aligned}
$$

where $L_{0 \mathrm{eff}}=\frac{1}{4\left(\mathrm{i} \rho^{0}\right)^{2}+\frac{1}{L^{2}}}$.

The planar contributions give rise to ordinary UV singularity. The non-planar contributions have two types of divergences $\mathrm{UV} / \mathrm{IR}$.

\section{Conclusion}

We have found two types of divergences UV/IR similar to those found in the contribution of the Ghost field loop [5]. We have given the contribution of the tadpole for the gauge propagator at one loop order. This contribution is (UV-IR) divergent as the loop of the theory calculated in [5]. Finally, the persistence of the ultravioletinfrared divergencies is interpreted as a further indication that UV-IR is essential property of Noncommutative field theories.

\section{References}

[1] S. Minwalla, M. Van Raamsdonk, N. Seiberg, JHEP 2000, 020 (2000); M. Van Raamsdonk, N.Seiberg, JHEP 2000, 035 (2000).

[2] Y. Liao, Nucl.Phys. B 713, 235 (2005).

[3] A. Hasimoto, N. Itzhaki, arXiv:hep-th/9907166, 1999.

[4] D. Klemm, S. Penati, L. Tamassia, arXiv:hepth/0104190, 2003.

[5] K. Zaibak, S. Kouadik, AIP Conf. Proc. 1444, 434 (2012). 